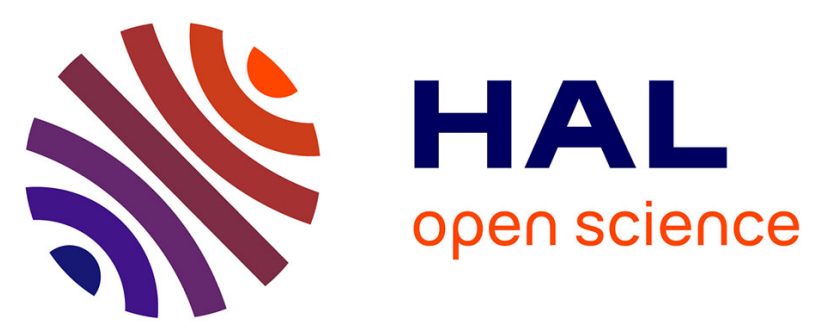

\title{
Atmospheric Pressure PhotoIonization versus Electrospray for the dereplication of highly conjugated natural products using molecular networks
}

\author{
Teo Hebra, Veronique Eparvier, David Touboul
}

\section{- To cite this version:}

Teo Hebra, Veronique Eparvier, David Touboul. Atmospheric Pressure PhotoIonization versus Electrospray for the dereplication of highly conjugated natural products using molecular networks. Journal of Chromatography A, 2020, 1630, pp.461533. 10.1016/j.chroma.2020.461533 . hal-03012257

\section{HAL Id: hal-03012257 \\ https://hal.science/hal-03012257}

Submitted on 18 Nov 2020

HAL is a multi-disciplinary open access archive for the deposit and dissemination of scientific research documents, whether they are published or not. The documents may come from teaching and research institutions in France or abroad, or from public or private research centers.
L'archive ouverte pluridisciplinaire HAL, est destinée au dépôt et à la diffusion de documents scientifiques de niveau recherche, publiés ou non, émanant des établissements d'enseignement et de recherche français ou étrangers, des laboratoires publics ou privés. 


\section{Atmospheric Pressure Photolonization versus Electrospray for \\ 2 the dereplication of highly conjugated natural products using \\ 3 molecular networks}

Teo Hebra, Véronique Eparvier, David Touboul*

Université Paris-Saclay, CNRS, Institut de Chimie des Substances Naturelles, UPR 2301, 91198, Gif-sur-Yvette, France.

* Corresponding author : David Touboul, david.touboul@cnrs.fr , phone number : +33 16982 3032

Abstract :

Natural products are sources of inspiration and reservoir of high valuable molecules. Recently, analytical tools based on liquid chromatography coupled to tandem mass spectrometry to generate molecular network became widely employed for dereplication. This strategy greatly accelerates the identification of known and structural hypothesis of unknown. Despite the availability of different ionization sources, alternatives to classical electrospray ionization (ESI), such as atmospheric pressure chemical ionization (APCI) or photoionization (APPI), have been neglected. In particular, APPI has been described for its ionization efficiency on non-polar molecules bearing no acid or basic groups. For that reason, we investigated APPI potential to generate molecular network and compare it to ESI on several criteria that are generation of ion species, sensitivity and signal-to-noise ratio (SNR) for different extracts rich in highly conjugated natural products. We first optimized APPI experimental conditions on crude extract from a fungus, Penicillium sclerotiorum (SNBCN111), producing polyketones belonging to the azaphilone family. Then we compared APPI and $\mathrm{ESI}$ on different fractions of the fungus and on two plant extracts, French Guyanese Swartzia panacoco (Aubl.) R.S. Cowan (arial parts) and Indian Cassia auriculata L. (leaves) containing phenolic compounds, such as flavonoids. While ESI generated more ion species and displayed a better sensitivity, APPI generated only protonated adduct and better SNR. Comparing ESI and APPI generated species on molecular network reveal that both strategies overlap for the majority of protonated ions.

Keywords: APPI, Molecular Network, Metabolomic, Azaphilone, Flavonoid 
Natural products are a source of inspiration as well as an important reservoir of molecules of high values in many economic fields such as medicine [1], nutrition [2], materials science [3] or cosmetics [4]. The analytical strategies used in metabolomics become gold standard in natural product research [5,6,7]. In particular, metabolomics accelerates the dereplication of complex mixtures, i.e. rapid annotation of already published compounds, for the discovery of undescribed products. One of the most widely used dereplication tool is the strategy based on liquid chromatography coupled to tandem mass spectrometry to generate molecular networks (LC-MS/MS-MN) [5]. Briefly, a data-dependent analysis (DDA) is performed throughout the chromatographic run. Cyclically, a MS1 scan is performed, then a fixed number of charged species, typically from 3 to 5 , will be sequentially fragmented and their respective MS2 spectra will be acquired. These fragmentation patterns will then be compared leading to the calculation of a cosine-score related to a structural homology distance. The generated distance matrix is finally used to generate the molecular networks (MNs). T-SNE algorithm can be finally implemented to improve the visualization of the MN. (gnps.ucsd.edu ) [8].

LC-MS/MS experiments to produce data for MN are usually performed with an electrospray ion source. More recently, methodologies to generate MNs from GC-EI-MS data have been introduced [9], (gnps.ucsd.edu). Nevertheless, alternative ion sources such as Atmospheric Pressure Chemical lonization (APCI) or Photoionization (APPI) have been largely neglected for such dereplication approaches. In particular, APPI has been previously described to be efficient for detection of non-polar or highly conjugated molecules bearing no acid or basic groups [10].

During APPI process, direct photoionization can occur if the ionization energy of the analyte is lower than the energy of photons emitted by the discharge lamp, typically 10.0 and $10.6 \mathrm{eV}$ for krypton. This phenomenon can be efficient on aromatic compounds for example. However, the major part of the analytes are not ionized or not sufficiently ionized by APPI leading to the introduction of a dopant molecule into the eluent. This electron or proton donor is usually a small organic molecule showing an ionization energy below $10 \mathrm{eV}$ such as anisole, toluene or acetone whereas [10] the common solvents, i.e. water and acetonitrile, are not photoionized due to ionization potential at 12.6 and $12.2 \mathrm{eV}$, respectively [11].

Some families of natural products have already been studied by APPI such as pentacyclic triterpene [12], mycotoxins [13], lipids [14,15,16], chlorophyll derivatives [17], flower absolutes [18] or polyphenols [19]. However, these studies were performed only from a diagnostic or quantification perspective and never in an unsupervised way. We choose to 
65 investigate two families of molecules likely to be studied with APPI: first,. highly conjugated 66 and colored polyketides (azaphilones and derivatives), with biological properties such as 67 antiparasitic [20] and antiviral [21] and proposed as potential natural food colorant [22,23] 68 and second, flavonoids, a molecular class with well-described antioxidant properties [24]. 69 Therefore, extracts from a microscopic fungus Penicillium sclerotiorum SNB-CN111, which species is mentioned to produce azaphilone and derivatives [25] and two plants, i.e. French Guyanese Swartzia panacoco (arial parts) and Indian Cassia auriculata, (leaves), both from the Fabales order, were investigated. Cassia auriculata is used in traditional Indian medicine and its extract possesses antioxidant properties [26]. We thus propose to compare APPI and ESI for the acquisition of MS2 data to generate MN in terms of number and type of detected species, sensitivity or MN information. 


\section{Material and method}

\subsubsection{Fungus material}

The fungus strain Penicillium sclerotiorum SNB-CN111 of strain library collection of ICSN (Sequencing allowed for strain identification by NCBI sequence comparison (Blastn ${ }^{\circledR}$ ); the sequence has been registered in the NCBI GenBank database (http://www.ncbi.nlm.nih.gov) under the registry number KJ023726) was cultivated on a large scale using 330 Petri dishes (14 cm diameter) at $28^{\circ} \mathrm{C}$ for 15 days on potato dextrose agar (PDA) medium (Dominique Dutscher SAS, Brumath, France). The culture medium containing the mycelium was cut into small pieces and macerated three time at room temperature with ethyl acetate (EtOAc) on a rotary shaker $(70 \mathrm{rpm})$ for $48 \mathrm{~h}, 24 \mathrm{~h}$ and $48 \mathrm{~h}$, respectively. The contents were extracted each time with $10 \mathrm{~L}$ of EtOAc using a separatory funnel. Insoluble residues were removed via filtration and the organic phase was washed three times with an equivalent volume of water $\left(\mathrm{H}_{2} \mathrm{O}\right)$, dried with anhydrous solid $\mathrm{Na}_{2} \mathrm{SO}_{4}$ and evaporated using a rotary evaporator under reduced pressure to yield a crude extract $(6.5 \mathrm{~g})$. The whole crude extract was fractionated by reversed-phase flash chromatography (Grace Reveleris, Grace, Maryland, USA) using a 120 gram C18 column and ultraviolet (UV) and evaporative light scattering detector (ELSD) detectors. A linear gradient of $\mathrm{H}_{2} \mathrm{O} /$ formic acid (99.9/0.1) - acetonitrile/formic acid (99.9/0.1) $\left(\mathrm{v} / \mathrm{v}, 95: 5\right.$ to $0: 100$ over $40 \mathrm{~min}$, flow rate at $\left.80 \mathrm{~mL} \cdot \mathrm{min}^{-1}\right)$ followed by another gradient of acetonitrile - methylene chloride ( $/ \mathrm{v}, 50: 50$ to 0:100 over $15 \mathrm{~min}$, flow rate at $80 \mathrm{~mL} \cdot \mathrm{min}^{-1}$ ) was set up to generate 11 fractions that were labeled F1 to F11.

\subsubsection{Plant material}

Swartzia panacoco stems and leaves were collected on the Couy River near Kourou (N 05'01.523', W 052.43.490'), French Guiana, in April 2014. This species is not a protected plant and can be collected without restriction at this location. A herbarium voucher (XC27) was deposited in Guyane IRD Herbarium (CAY). Plant samples were dried (room temperature, $10 \%$ air relative humidity) and finely powdered. Two sample of $300 \mathrm{~g}$ of stems and leaves were extracted at room temperature by maceration using $3 \times 1 \mathrm{~L}$ of EtOAc or $\mathrm{MeOH}$ providing four different extracts after evaporation of the solvents.

Cassia auriculata leaves (1 g, Aroma-Zone, Paris, France) from India were macerated during $24 \mathrm{~h}$ at room temperature on a rotary shaker $(70 \mathrm{rpm})$ with either methanol, ethyl acetate or methylene chloride $(200 \mathrm{~mL})$. Insoluble residues were removed via filtration and the organic phase was washed three times with an equivalent volume of $\mathrm{H}_{2} \mathrm{O}$ using a separatory funnel, dried with anhydrous solid $\mathrm{Na}_{2} \mathrm{SO}_{4}$ and evaporated using a rotary evaporator under reduced 
pressure to yield a crude mixture. The first equivalent of water from EtOAc extract was kept

112 to be dried leading to $6 \mathrm{mg}$ for $\mathrm{H}_{2} \mathrm{O}$ fraction, $82 \mathrm{mg}$ for $\mathrm{MeOH}, 62 \mathrm{mg}$ for EtOAc and $56 \mathrm{mg}$ 113 for $\mathrm{CH}_{2} \mathrm{Cl}_{2}$.

\subsection{Data acquisition}

Fractions from Penicillium sclerotiorum SNB-CN111 or crude extract from Cassia auriculata leaves and Swartia panacoco leaves and stems were prepared at $1 \mathrm{mg}^{-1} \mathrm{ml}^{-1}$ in methanol and filtered on $0.45 \mu \mathrm{m}$ PTFE membrane. LC-MS/MS experiments were performed with a 1260 Prime HPLC (Agilent Technologies, Waldbronn, Germany) coupled with an Agilent 6540 QToF (Agilent Technologies, Waldbronn, Germany) tandem mass spectrometer. LC separation was achieved with an Accucore RP-MS column $(100 \times 2.1 \mathrm{~mm}, 2.6 \mu \mathrm{m}$, Thermo Scientific, Les Ulis, France) with a mobile phase consisting of $\mathrm{H}_{2} \mathrm{O} /$ formic acid (99.9/0.1) (A) and acetonitrile with $0.1 \%$ formic acid (B). The column oven was set at $45{ }^{\circ} \mathrm{C}$. Compounds were eluted at a flow rate of $0.4 \mathrm{~mL} \cdot \mathrm{min}^{-1}$ with a gradient from $5 \% \mathrm{~B}$ to $100 \% \mathrm{~B}$ in $20 \mathrm{~min}$ and then $100 \%$ B for $3 \mathrm{~min}$. Injection volume was fixed at $5 \mu \mathrm{L}$ for all analyses. For electrospray ionization source, mass spectra were recorded in positive ion mode with the following parameters: gas temperature $325^{\circ} \mathrm{C}$, drying gas flow rate $10 \mathrm{~L} . \mathrm{min}^{-1}$, nebulizer pressure 30 psi, sheath gas temperature $350{ }^{\circ} \mathrm{C}$, sheath gas flow rate $10 \mathrm{~L} \cdot \mathrm{min}^{-1}$, capillary voltage 3500 $\mathrm{V}$, nozzle voltage $500 \mathrm{~V}$, fragmentor voltage $130 \mathrm{~V}$, skimmer voltage $45 \mathrm{~V}$, Octopole 1 RFVoltage $750 \mathrm{~V}$. The collision energy was fixed at $30 \mathrm{eV}$ for all the MS/MS experiments. For ESI, internal calibration was achieved with two calibrants $(\mathrm{m} / \mathrm{z} 121.0509$ and $\mathrm{m} / \mathrm{z}$ 922.0098) providing a high mass accuracy better than $3 \mathrm{ppm}$. For atmospheric pressure photoionization source mass spectra were recorded in positive ion mode with the following starting parameters for the optimization: gas temperature set at $325^{\circ} \mathrm{C}$, vaporizer set at 300 ${ }^{\circ} \mathrm{C}$, capillary voltage set at $2500 \mathrm{~V}$, fragmentor voltage at $130 \mathrm{~V}$. The dopant flow was set at $30 \mu \mathrm{L}$ per minute. For the analysis once optimized, APPI parameters were set to gas temperature set at $325{ }^{\circ} \mathrm{C}$, vaporizer set at $300{ }^{\circ} \mathrm{C}$, capillary voltage set at $2000 \mathrm{~V}$, fragmentor voltage at $130 \mathrm{~V}$. The dopant was acetone and its flow was set at $10 \mu \mathrm{L} \cdot \mathrm{min}^{-1}$. External calibration was achieved with LC/MS tuning mix, for APCI/APPI (Agilent Technologie, Massy, France) and mass accuracy was better than $5 \mathrm{ppm}$ at $\mathrm{m} / \mathrm{z} 383$. The data-dependent MS/MS events were acquired for the five most intense ions detected by fullscan MS (maximum of five precursors per cycle of $1.2 \mathrm{~s}$ ) above an absolute threshold of 1000 counts. Selected parent ions were fragmented at a fixed collision energy of $30 \mathrm{eV}$ and with an isolation window of $1.3 \mathrm{amu}$. The mass range of the precursor and fragment ions was set as $m / z 200-1000$.

\subsection{Data processing and analysis}


The data files were converted from the .d (Agilent Technologies, Massy, France) standard data format to .mzXML format using the MSConvert software, part of the ProteoWizard package 3.0 [28]. All .mzxml were processed using MZmine2v51 as previously described [29]. The mass detection was realized with MS1 noise level at 1000 and MS2 noise level at 0 . The ADAP chromatogram builder was employed with a minimum group size of scans of 3 , a group intensity threshold of 1000 , a minimum highest intensity of 1000 , and $\mathrm{m} / \mathrm{z}$ tolerance of 0.008 (or $20 \mathrm{ppm}$ ). Deconvolution was performed with the ADAP wavelets algorithm according to the following settings: $S / N$ threshold=10, minimum feature height $=1000$, coefficient/area threshold=10, peak duration range0.01-1.5min, RT wavelet range 0.00 $0.04 \mathrm{~min}$. MS2 scans were paired using an $\mathrm{m} / \mathrm{z}$ tolerance range of $0.05 \mathrm{Da}$ and RT tolerance range of $0.5 \mathrm{~min}$. Isotopologues were grouped using the isotopic peak grouper algorithm with an $\mathrm{m} / \mathrm{z}$ tolerance of 0.008 (or $20 \mathrm{ppm}$ ) and a RT tolerance of $0.2 \mathrm{~min}$. Peaks were filtered using Feature list row filter, keeping only peaks with MS2 scan (GNPS). Adduct identification, i.e. sodium- or potassium-cationized species, was performed on the peak list with a retention time tolerance of $0.1 \mathrm{~min}$, an $\mathrm{m} / \mathrm{z}$ tolerance of 0.008 or $20 \mathrm{ppm}$, and a maximum relative peak height of $150 \%$. Complex search, such as dimers, was performed with a retention time tolerance of $0.1 \mathrm{~min}$, an $\mathrm{m} / \mathrm{z}$ tolerance of 0.008 or $20 \mathrm{ppm}$ and a maximum relative peak height of $150 \%$. Peak alignment was performed using the join aligner with an $\mathrm{m} / \mathrm{z}$ tolerance of 0.008 (or $20 \mathrm{ppm}$ ), a weight for $\mathrm{m} / \mathrm{z}$ at 20 , a retention time tolerance of $0.2 \mathrm{~min}$ and a weight for RT at 50 . The MGF file and the metadata were generated using the export/submit to GNPS option.

Cosine score were calculated on MetGem 1.3 software [8], MetGem parameters. MS2 spectra were window-filtered by choosing only the top 6 peaks in the $\pm 50 \mathrm{Da}$ window throughout the spectrum. The data was filtered by removing all peaks in the $\pm 17 \mathrm{Da}$ range around the precursor $\mathrm{m} / \mathrm{z}$. The $\mathrm{m} / \mathrm{z}$ tolerance windows used to find the matching peaks was set to $0.02 \mathrm{Da}$ and cosine score were kept in consideration for spectra sharing 2 matching peaks at least. The number of iterations, perplexity, learning-rate and early exaggeration parameters were set to $5000,25,200$ and 12 , respectively.

Annotations of molecules in MN were made using "Find standards in library" or "Find analogs in library" functions of MetGem. Queries were made against Massbank NA (https://mona.fiehnlab.ucdavis.edu), GNPS Public Spectral Library (https://gnps.ucsd.edu), NIST14 Tandem (https://www.nist.gov), NIH Natural Product (https://www.nih.gov) and MSDial (http://prime.psc.riken.jp/compms) libraries with absolute $\mathrm{m} / \mathrm{z}$ tolerance of $0.02,4$ minimum matched peaks and minimal cosine score of 0.5 . 
180 To annotate molecules with high confidence in MN using standard search in library, both 181 exact mass, comparison of $\mathrm{MS}^{2}$ spectra and previous report of molecules within genera or 182 species are taken into account.

183 Ion intensity and signal-to-noise ratio (SNR) for diagnostic ions were extracted with 184 MassHunter software (AgilentTechnologie, Massy, France).

185 Figures were generated using $\mathrm{R}$ and related packages (ggplot2, VennDiagram, Rcolorbrewer 186 and gridextra). 


\subsection{Parameter optimization}

190 In order to optimize APPI conditions, several parameters were investigated such as vaporizer temperature, the capillary voltage, the dopant chemistry and flow rate. The influence of these parameters on ionization and signal-to-noise ratio was then evaluated. Therefore, we selected several ions belonging to the azaphilone family from a crude extract from Penicillium sclerotiorium SNB-CN111. Five of them were the most abundant species, i.e. ochrephilone $\left([\mathrm{M}+\mathrm{H}]^{+}, \mathrm{m} / \mathrm{z}\right.$ 383.1860); sclerotioramine $\left([\mathrm{M}+\mathrm{H}]^{+}, \mathrm{m} / \mathrm{z} 390.1472\right)$; sclerotiorin $\left([\mathrm{M}+\mathrm{H}]^{+}, m / z\right.$ 391.1312); geumsanol $\mathrm{B}\left([\mathrm{M}+\mathrm{H}]^{+}, \mathrm{m} / z\right.$ 417.1914); isochromophilone $\mathrm{VI}\left([\mathrm{M}+\mathrm{H}]^{+}\right.$, $m / z$ 434.1735)) and three were less abundant ones (unknown([M+H] $]^{+}, m / z$ 353.1967), isorotiorin( $\left.[\mathrm{M}+\mathrm{H}]^{+}, m / 385.2015\right)$ and unknown $\left([\mathrm{M}+\mathrm{H}]^{+}, \mathrm{m} / z\right.$ 451.1525). Formation of radical ion $[\mathrm{M}]^{*+}$ is expected on APPI experiment but none were observed with acetone as dopant, and less than $2 \%$ with toluene (supplementary figure 1). It can be explained by several of our experimental condition: protic solvent like water are known to support apparition of $[\mathrm{M}+\mathrm{H}]^{+}$ [30], acetone as dopant is described to enhance the formation of protonated species [31]. As for toluene, working with acetonitrile inhibit toluene $[\mathrm{M}]^{*+}$ formation, thus leading to major $[\mathrm{M}+\mathrm{H}]^{+}$population [32]. Optimizations of drying gas temperature, capillary voltage and dopant flow are illustrated below with acetone as a dopant.

\subsubsection{Effect of the vaporizer temperature}

208

209

210

Because ionization under APPI happens in a vapor like state, vaporizer temperature is a key factor in order to maximize the ion intensity or the signal-to-noise ratio (SNR). A low vaporizer temperature could not vaporize enough mobile phase leading to signal dilution whereas too high temperature could lead to molecular degradation. A temperature range between 200 to $450^{\circ} \mathrm{C}$ with $50^{\circ} \mathrm{C}$ step was thus investigated for acetone at a flow rate of 10 $\mu L \cdot \min ^{-1}$.

Two types of behavior can be described based on figure 1. For 5 over 8 diagnostic ions $(\mathrm{m} / \mathrm{z}$ 353.1967, 390.1472, 417.1914 434.1735 and 451.1525), both intensities and SNR are the lowest at $200^{\circ} \mathrm{C}$ then reach a plateau between 250 and $350^{\circ} \mathrm{C}$ then dramatically decrease. For the other 3 diagnostic ions ( $\mathrm{m} / \mathrm{z} 383.1860,385.2015$ and 391.1312), a slight intensity decrease or a plateau is occurring from 200 to $450{ }^{\circ} \mathrm{C}$. Therefore, a vaporizer temperature at $300^{\circ} \mathrm{C}$ was chosen for the following optimization steps because this source condition led to 
the best intensity and SNR for 6 over 8 diagnostic ions while being acceptable for the two

221 last ones (figure 1a, a1b).

\subsubsection{Effect of the capillary voltage}

The capillary voltage can affect the transmission efficiency of the ions at the entrance of the mass spectrometer and is rather independent to the vaporizer temperature. A voltage range from 2000 to $3500 \mathrm{~V}$ by $500 \mathrm{~V}$ step was scanned. As depicted on Figure 2, this parameter has no significant effect on the final intensity or SNR of the diagnostic ions and was optimal at 2000 V. This value was chosen for the following optimization steps.

\subsubsection{Effect of the dopant flow rate}

231

232

233

234

When direct photoionization of compound is not efficient, the addition of a dopant is necessary. The dopant will exchange its charge or radical hydrogen to the analyte [33]. In the case of acetone, the main ionization process is the formation of protonated acetone followed by a proton transfer to the analyte. One important reported parameter in the literature is the flow rate of the dopant $[34,35]$. If the dopant quantity is too low, the ionization process will not be optimal whereas a too high quantity will lead to increasing noise signals. The dopant flow rate has been empirically established to $5-10 \%$ of the total flow rate. Therefore, typical dopant flow rates from 10 to $50 \mu \mathrm{L} \cdot \mathrm{min}^{-1}$ were investigated (figure $3 \mathrm{a}, 3 \mathrm{~b}$ ).

Without dopant, proportion of $[\mathrm{M}]^{+\cdot}$ significantly increase (Supplementary figure 2) suggesting that highly conjugated azaphilone are subject to direct photoionization but both signal intensity and SNR for all diagnostic ions are drastically increased when introducing the dopant. The dopant flow rates had no significant impact on the intensity of the 8 diagnostic ions (figure 3a) whereas increasing flow rate of the dopant decreases the SNR (figure 3b). Thus the optimal value is $10 \mu \mathrm{L} \cdot \mathrm{min}^{-1}$ for acetone representing $2.5 \%$ of the eluent flow rate. This value is slightly under described ones [34] 5 to $10 \%$ of eluent flow rate but it could be related to the use of acetone instead of toluene.

\subsubsection{Effect of the dopant chemistry}


Acetone, anisole and toluene are classical dopants handled to increase APPI efficiency. We excluded anisole as a dopant from the analysis due to several intense ions at $m / z 206.1666$, 230.0937, 263.0917, 530.4691 and 647.4576 throughout the chromatographic time saturating the signal. The same optimization procedure as for acetone was operated for toluene (supplementary figure 3 ). The selected parameters for toluene were as follows: vaporizer temperature at $350{ }^{\circ} \mathrm{C}$, capillary voltage at $2500 \mathrm{~V}$ and dopant flow at $30 \mu \mathrm{L} \cdot \mathrm{min}^{-1}$. The optimized flow rate of toluene was higher compared to acetone as previously described in the literature [34].

Acetone as a dopant outperforms toluene for the intensity of 6 diagnostic ions and displays same intensities for the two others ( $\mathrm{m} / \mathrm{z} 417$ and 434) (figure 4a). Regarding SNR, acetone fully exceeds toluene by a factor of 3 (figure $4 b$ ). Some author reported this phenomenon and suggest that toluene impurities could explain this discrepancy [35]. Based on this experiment, acetone was kept as a dopant for further experiments using the previously optimized ionization conditions.

3.2 Comparison of ESI and APPI for the dereplication of Penicillium sclerotiorum SNBCN111

In order to evaluate APPI-MS for dereplication of complex mixture of natural products by molecular networking $[8,28,36]$, Penicillium sclerotiorum SNB-CN111 crude extract has been fractionated by polarity. Nine fractions were thus analyzed by reversed-phase liquid chromatography couple to tandem mass spectrometry (LC-MS/MS) in ESI and APPI positive modes. Then, the data have been processed by MZmine2 and MetGem software to construct molecular network containing data from both ionization sources.

\subsubsection{Qualitative analysis of ESI and APPI analysis}

Chromatographic run with ESI source produce significantly more ion signals than APPI (Figure 5a). Indeed, over the 9 analyzed fractions, ESI generated 3152 nodes (precursor ion / retention time couples) for which a $\mathrm{MS}^{2}$ was acquired. This is 4.2 time more than APPI (747). About $15 \%$ (462) of ESI signals were annotated by MZmine2 fragment search algorithm as $[\mathrm{M}+\mathrm{H}]^{+},[\mathrm{M}+\mathrm{Na}]^{+},[\mathrm{M}+\mathrm{K}]^{+},[2 \mathrm{M}+\mathrm{H}]^{+},[2 \mathrm{M}+\mathrm{Na}]^{+},\left[\mathrm{M}+\mathrm{M}^{\prime}+\mathrm{H}\right]^{+}$or $[\mathrm{M}+\mathrm{M}+\mathrm{Na}]^{+}$of the same molecule M. A manual search within the most intense ions, as well as the clustering of ESI nodes in the t-SNE representation, suggests that this number is largely underestimated. This phenomenon is common within ESI experiment and manual inspection is often 
mandatory to avoid misinterpretation or redundant information. On the contrary, APPI ion signals were strictly annotated as protonated species. Common features between ESI and APPI are estimated to be 497 corresponding to $66 \%$ of APPI signals. Moreover, these common features are related to ion population of high intensity (Figure 5b, 5c) and can be employed for reducing the redundant information from non-protonated species. Furthermore, ESI led to the detection of 125 features present in each sample and in the blank (pure methanol) whereas APPI only exhibited 7 interferences from the blank, i.e. pure methanol.

An intensity threshold value of 100000 , corresponding to two orders of magnitude between the most and less intense feature, was added to simplify the reading of the molecular networks (figure 5c,6a) and get rid of very low intensity ions. The dataset is finally composed of 691 ions, i.e. 374 APPI features, 643 ESI ones which 102 of them are non-protonated species and 326 are common between the two ionization methods (figure $6 \mathrm{~b}$ ). This clearly highlights the fact that major differences between ESI and APPI features are for low abundant species and non-protonated species.

It is interesting to note that the species present in the south of the t-SNE MN is mainly constituted of bimolecular species such as $[2 \mathrm{M}+\mathrm{H}]^{+},[2 \mathrm{M}+\mathrm{Na}]^{+},\left[\mathrm{M}+\mathrm{M}{ }^{\prime}+\mathrm{H}\right]^{+}$or $\left[\mathrm{M}+\mathrm{M}^{\prime}+\mathrm{Na}\right]^{+}$ (figure 6a). To understand better the differences of ionization between several scaffold of azaphilone such as ochrephilone, isochromophilone VI or geumsanol B, MN annotation is provided in Figure 7 and led to the annotation of 16 molecular classes using dereplication tools such as $\mathrm{MS}^{2}$ database search, the Natural Product Atlas [37] and a review of literature on Azaphilone and Penicillium sclerotiorum or multicolor. [38,39]

303

304

Several signals have been directly annotated through $\mathrm{MS}^{2}$ database comparison, the database compound with the highest homology cosine score was used for annotation, such as isochromophilone VI (cluster1, cosine score $=0.85$ ), sclerotioramine (cluster2, cosine score $=0.97$ ), ochrephilone (cluster4, cosine score $=0.92$ ), hypocrellone A (cluster 6 , cosine score $=0.76$ ), geumsanol $\mathrm{C}$ (cluster 7 , cosine score $=0.59$ ), several diketopiperazine (cluster 8, CycloPhe-Pro, cosine score $=0.97$, CycloPheLeu, cosine score $=0.92$, CycloPheHydroxyPro, cosine score $=0.80$ ), monoelaidin (cluster 9, cosine score $=0.86$ ), isochromophilone IV (cluster 13, cosine score $=0.75$ ), sclerotiorine (cluster 14 , cosine score $=0.82$ ), lauryl ethanolamide (cluster 15 , cosine score $=0.87$ ). Ergosterol derivatives (cluster 10) and sclerotioramine analogs (cluster 12) were found through analogs search into $\mathrm{MS}^{2}$ databases. Their scaffolds were common and redundant over several nodes present in their respective cluster. Geumsanol C (cluster 7) annotation with a homology cosine score from databases of 0.59 was low for a highly confident annotation, we decided to include it because we manually found other evidence that increased our confidence: its chlorinated 
analog is present within the same cluster and also annotated through databases search with the highest compound hit of 0.66. . Finally, Peniazaphilone A (cluster 3,0 ppm from calculated mass), Geumsanol B (cluster $5,+1.7$ ppm from calculated mass) are dereplicated using the structural cluster search of Natural Product Atlas. Most of these molecule share a common scaffold and belongs to the azaphilone family. Nevertheless, there is a diversity of substructure that can impact ionization: addition of hydroxyl (Geumsanol B, Geumsanol C, Hypocrellone A), a lactone ring (Peniazaphilone A, Ochrephilone, Geumsanol B), substitution of the oxygen by a nitrogen (Isochromophilone VI, Sclerotioramine, Peniazaphilone A). This is why we decided to make a dilution range of the fractions from $1000 \mu \mathrm{g} \cdot \mathrm{mL}^{-1}$ to $25 \mu \mathrm{g} \cdot \mathrm{mL}^{-1}$ in order to evaluate sensitivity and SNR for APPI compare to ESI (figure 8b-8k).

ESI outperformed APPI in term of ionization for 4 out of 6 species (Ochrephilone (I), Sclerotiorine (II), Sclerotioramine (III), Isochromophilone VI (IV)) and displayed similar ionization to APPI for the two others (Geumsanol B (V) and 5-chloroisorotiorin (VI)) at 25 and $100 \mu \mathrm{g} \cdot \mathrm{ml}^{-1}$. At $250 \mu \mathrm{g} \cdot \mathrm{ml}^{-1} \mathrm{ESI}$ performed better for 3 out of 6 ions (Ochrephilone (I), Sclerotioramine (III) and Isochromophilone VI (IV)). This is not the case anymore for Sclerotiorine (II) as APPI and ESI equally perform, as well for Geumsanol B (V) and 5chloroisorotiorin ( $\mathrm{VI})$. Despite a saturation phenomenon at $1000 \mu \mathrm{g} \cdot \mathrm{ml}^{-1}$, it is interesting to note that only the 2 nitrogen molecules (Sclerotioramine (III) and Isochromophilone VI (IV)) are better ionized by ESI (figure $8 \mathrm{a}-8 \mathrm{~g}$ ).

APPI leads to higher SNR for 3 molecules out of 6 (Ochrephilone (I), Isochromophilone VI (IV), Geumsanol B (V)) compared to ESI, similar values for (5-chloroisorotiorin (f)) and lower for 2 molecules (Sclerotiorine (II), Sclerotioramine (III)) at $25 \mu \mathrm{g} \cdot \mathrm{ml}^{-1}$. The capability of APPI to achieve higher SNR compared to ESI increases for the higher concentration: 5 out of 6 molecules (Ochrephilone (I), Sclerotiorine (II), Sclerotioramine (III), Isochromophilone VI (IV) and 5-chloroisorotiorin (VI)) display better SNR value with APPI at $100 \mu \mathrm{g} \cdot \mathrm{ml}^{-1}$, for example (figure $8 \mathrm{a}, 8 \mathrm{~h}-8 \mathrm{k}$ ).

Overall, ESI and APPI displayed different but complementary features for the analysis of the specialized metabolism of Penicillium sclerotiorum. While ESI displays better sensitivity overall, APPI showed better SNR and produces only protonated species leading to an easier data interpretation. Similar results were obtained on other natural product families, indicating that overall, APPI is less susceptible to matrix effect [40,41]. Once data from ESI and APPI are combined together, they allow a highly valuable molecular snapshot of a complex sample.

\footnotetext{
3.3 Comparison of ESI and APPI for the analysis of flavonoid containing plants.
} 
To extend the scope of this study, we have chosen to investigate another class of natural product, i.e. flavonoids in crude plant extracts. Swartia panacoco and Cassia auriculata specialized metabolites were extracted with solvent of different polarity (water, methanol, ethyl acetate and dichloromethane). The extract was thus analyzed by LC-MS/MS using either ESI or APPI in positive mode.

\subsubsection{Qualitative analysis of ESI and APPI analysis}

In accordance with the results previously described with Penicillium sclerotiorum fractions, LC-MS with ESI source produces significantly more ion signals than APPI (Figure 9a, 9c). Indeed, over the analyzed 8 crude extract (2 plants and 4 extraction solvents each), ESI generated 3560 nodes (precursor ion / retention time couples) for which a $\mathrm{MS}^{2}$ was acquired. This is 5.2 time more nodes than APPI (675). About 6\% (222) of ESI signals were annotated by $\mathrm{MZmine} 2$ fragment search algorithm as $[\mathrm{M}+\mathrm{H}]^{+},[\mathrm{M}+\mathrm{Na}]^{+},[\mathrm{M}+\mathrm{K}]^{+},[2 \mathrm{M}+\mathrm{H}]^{+},[2 \mathrm{M}+\mathrm{Na}]^{+}$, $\left[\mathrm{M}+\mathrm{M}^{\prime}+\mathrm{H}\right]^{+}$or $\left[\mathrm{M}+\mathrm{M}^{\prime}+\mathrm{Na}\right]^{+}$of the same molecule $\mathrm{M}$. On the contrary, APPI ion signals were strictly annotated as protonated species. Common features between ESI and APPI are 384 corresponding to $56 \%$ of APPI signals. Moreover, these common features are related to ion population of high intensity (Figure $9 b$ ) and can be employed for reducing the redundant information from non-protonated species. Furthermore, ESI led to the detection of 152 features present in each sample and in the blank (pure methanol) whereas APPI only exhibited 9 interferences from the injection solvent. All these observations are in perfect accordance with results obtained on Penicillium sclerotiorum.

Following the same procedure as before, an intensity threshold was added to simplify the reading of the molecular networks and get rid of very low intensity ions. This time value of 50000 has been defined, to keep to two orders of magnitude between the most and less intense feature. The dataset is finally combining 898 nodes, i.e. 295 APPI features, 870 ESI ones which 49 of them are non-protonated species and 267 are common between the two ionization methods (figure 10a, 10b). Once again major differences between ESI and APPI are concentrated on low abundant or non-protonated species.

It is interesting to note that the west of the t-SNE MN is mainly constituted of high intensity nodes produced by both APPI and ESI. The nodes specific to APPI are concentrated into two clusters (in the south west and the south east) and one isolated node in the north. MN annotation is provided in Figure 11 to better understand the specificity of APPI and ESI, and to verify if our hypothesis considering APPI as a technic of interest to search for flavonoid in a complex extract. It led to the annotation of 14 clusters using MS $^{2}$ database search dereplication tools. Cluster1 was annotated as glycosylated flavonoids (quercetin-4O- 
glucoside, $m / z$ 465.0988, cosine score $=0.79$ ), cluster 2 as flavonoids (3-O-methylquercetin, $m / z 317.0628$, cosine score $=0.84$, luteolin, $m / z 287.0724$, cosine score $=0.88$ and catechin, $m / z$ 291.0848, cosine score $=0.87$ ), cluster3 as Pheophorbide $A(m / z 593.275$, cosine score $=0.75$ ), cluster 4 as Pheophytin $A$ analogs (Pheophytin $A, m / z 871.5748$, cosine score $=0.69$ ), cluster 5 as ceramide (analog search on the whole cluster), cluster 6 as phytosphingosine $(\mathrm{m} / \mathrm{z} 318.2977$, cosine score $=0.73)$, cluster 7 as phosphatidylcholine (Lyso-PC(16:0), $m / z$ 496.3387, cosine score $=0.87$ ), cluster 8 as adenosine and derivatives (adenosine, $\mathrm{m} / \mathrm{z}$ 268.1211, cosine score $=0.83$ ), cluster 9 as phtalates (dioctyl phtatalate, $\mathrm{m} / \mathrm{z}$ 391.2822, cosine score $=0.80$ ), cluster 10 as monoglyceride (glycerol 1 -stearate, $\mathrm{m} / \mathrm{z}$ 359.313 , cosine score $=0.89$ ), cluster 11 as fatty acids (octadecadienoic acid, $\mathrm{m} / z$ 295.225, cosine score $=0.66$ ), cluster 12 was pentacyclic triterpene (glycyrrhetinic acid, $m / z 471.3433$, cosine score $=0.71$ ), cluster13 as diglyceride/triglyceride (analogs search on the whole cluster) and cluster 14 as fatty acid amide (linoleyl-ethanolamide, $m / z 324.287$, cosine score $=0.79$ ).

A single cluster at the extreme east, and annotated as polyethylene glycol (PEG) derivatives, has been cut off because it was too far away and took away legibility. From the two clusters constituted of APPI-only feature, one is constituted of ceramide analogs while the other seems to be a subclass of pentacyclic triterpene. Moreover, the APPI generated ion intensity of pentacyclic triterpenes is directly depending to their polarity (Supplementary figure 4); the less polar, the better the intensity was. As previously described in the literature, highly apolar pentacyclic triterpenes are well suited for APPI analysis in the positive mode [12].

Because our aim was to characterize and compare APPI performances over ESI on highly conjugated molecules, we extracted both intensity and SNR and intensity for a panel of 6 molecules representative of flavonoid clusters. Two molecule from the purple sub-cluster (3O-methylquercetin, cosine score $=0.84$, and formononetin, cosine score $=0.84$, cluster 2$), 1$ molecule from the north pink sub-cluster (luteolin, cosine score $=0.88$, cluster 2 ), 2 molecule from the brown sub-cluster (Epigallocatechin, cosine score $=0.7$ and Catechin, cosine score $=0.87$, cluster 2) and one molecule from the glycosylated flavonoid green sub-cluster (Quercetin-4O-glucoside, cosine score $=0.79$, cluster 1 ) of the t-SNE representation (Figure 11).

For 3-o-methylquercetin (I), APPI outperforms ESI both in intensity and SNR. ESI and APPI displays equal sensitivity for Formononetin (II) and Luteolin (III) and similar SNR for Formononetin (II) while SNR of Luteolin (III) is better for ESI. For the last 3 diagnostic molecules (Epigallocatechin (IV), Catechin (V) and Quercetin-4-o-glucoside (VI)) ESI operates much better than APPI for both intensity and SNR. The global behavior is following 
422 the retention times and thus the polarity as described for pentacyclic triterpenes. This results

423 confirmed that APPI can be highly performant for the ionization of apolar molecules, as

424 described within several lipids classes [14], leading to highly informative molecular networks. 
427 APPI-MS parameters were optimized for the analysis of highly conjugated and apolar 428 compounds from a fungus crude extract with an emphasis on sensitivity and SNR. While 429 ESI-MS generates more species and displayed a better sensitivity overall, APPI-MS only 430 generates protonated species, displays better SNR and generated less interfering species. 431 These conclusions were extended by the study of arial parts extracts from French Guyanese 432 Swartzia panacoco and Indian Cassia auriculata, especially for flavonoid and pentacyclic 433 triterpene families. Using complementarity between APPI-MS and ESI-MS, a strategy for the 434 analysis of complex natural extracts by molecular network could start with an APPI-MS 435 analysis to characterize and annotate ions. This will avoid redundant signals because APPI436 MS generate only protonated species with high SNR. Then a second batch of analysis could 437 be performed using ESI-MS in order to detect and annotate low abundance ions thanks to 438 the better sensitivity of ESI-MS.

\section{Acknowledgements}

441 Teo Hebra thanks the ministère de l'Enseignement supérieur, de la Recherche et de 442 I'Innovation (MESRI) for his PhD fellowship. This work was supported by an "Investissement 443 d'Avenir" grant (CEBA, ref ANR-10-LABX-0025) managed by the French National Research 444 Agency (ANR). 
Figure legends:

450 Figure 1: Effect on (a) intensity or (b) signal-to-noise ratio (SNR) of vaporizer temperature for 4518 ions from Penicillium sclerotiorum SNB-CN111 crude extract. Logarithmic scale.

452 Figure 2: Effect on(a) intensity or (b) signal-to-noise ratio (SNR) of capillary voltage for 8 ions 453 from Penicillium sclerotiorum SNB-CN111 crude extract. Logarithmic scale.

Figure 3: Effect on(a) intensity or (b) signal-to-noise ratio (SNR) of acetone dopant flow for 8 ions from Penicillium sclerotiorum SNB-CN111 crude extract. Logarithmic scale.

Figure 4: Effect on(a) intensity or (b) signal-to-noise ratio (SNR) of dopant chemistry for 8 ions from Penicillium sclerotiorum SNB-CN111 crude extract. Logarithmic scale.

Figure 5: (a) lon distribution from Penicillium sclerotiorum SNB-CN111 fractions between ESI and APPI; (b) their respective intensity their (c) t-SNE representation constructed on $\mathrm{MS}^{2}$ homology the size of nodes is related to their intensity.

Figure 6: Penicillium sclerotiorum SNB-CN111 fractions: (a) t-SNE representation constructed on $\mathrm{MS}^{2}$ homology and (b) Ion repartition between ESI and APPI. An intensity threshold value of 100000 is applied to the dataset, the size of nodes is related to their intensity.

Figure 7: Structural annotation of the molecular network related to Penicillium sclerotiorum SNB-CN111 fractions. Contaminants are composed of polydimethylsiloxane (PDMS) from vial septum (hit on databases).

Figure 8: (a) Diagnostic molecules (Ochrephilone (I), Sclerotiorine (II), Sclerotioramine (III), Isochromophilone VI (IV), Geumsanol B (V) and 5-chloroisorotiorin (VI)). Ion counts (b, c, d, e, f, g) and SNR (h, I, j, k) for the respective protonated species. Logarithmic scale.

Figure 9: (a) Ion distribution of specialized metabolites from Swartia panacoco and Cassia auriculata crude extracts analyzed by ESI and APPI, (b) their respective intensity (logarithmic scale), and (c) t-SNE representation constructed on $\mathrm{MS}^{2}$ homology, the size of nodes is related to their intensity.

Figure 10: (a) t-SNE representation -from Swartia panacoco and Cassia auriculata crude extracts- constructed on $\mathrm{MS}^{2}$ homology, the size of nodes is related to their intensity. (b) lon repartition between ESI and APPI. An intensity threshold value of 50000 is applied to the dataset. 
479 Figure 11: Annotation of the molecular network (t-SNE view) from 8 extracts of two plant 480 leaves (Swartia panacoco and Cassia auriculata). 1: Glycosylated flavonoid, 2: Flavonoid, 481 3: Pheophorbide A, 4: Pheophytin A analogs, 5: Ceramide, 6: Phytosphingosine, 482 7: Phosphatidylcholine, 8: Adenosine and derivative, 9: Phtalate, 10: Monoglyceride, 11: 483 Fatty acid, 12: Pentacyclic triterpene, 13: Diglyceride/Triglyceride, 14: Fatty acid amide.

484 Figure 12: (a) Diagnostic molecules (3-O-methylquercetin (I), Formononetin (II), Luteolin (III), 485 Epigallocatechin (IV), Catechin (V) and Quercetin-4-O-glycoside (VI)), Ion counts (b) and 486 SNR. (c) for the respective protonated species Logarithmic scale.

487 
490 [1] Newman, D.J., Cragg, G.M., 2016. Natural Products as Sources of New Drugs from 1981

491

492

493

494

495

496

497

498

499

500

501

502

503

504

505

506

507

508

509

510

511

512

513

514

515

516

517

518

519

520

521

522

523

524

525

526

527

528

529

530

531

532

533

534

535

536 to 2014. J. Nat. Prod. 79, 629-661. https://doi.org/10.1021/acs.jnatprod.5b01055

[2] Panche, A.N., Diwan, A.D., Chandra, S.R., 2016. Flavonoids: an overview. J Nutr Sci 5, e47. https://doi.org/10.1017/jns.2016.41

[3] Zhu, Y., Romain, C., Williams, C.K., 2016. Sustainable polymers from renewable resources. Nature 540, 354-362. https://doi.org/10.1038/nature21001

[4] Lourith, N., Kanlayavattanakul, M., 2009. Natural surfactants used in cosmetics: glycolipids. International Journal of Cosmetic Science 31, 255-261. https://doi.org/10.1111/j.1468-2494.2009.00493.x

[5] Wolfender, J.-L., Litaudon, M., Touboul, D., Queiroz, E.F., 2019. Innovative omics-based approaches for prioritisation and targeted isolation of natural products - new strategies for drug discovery. Nat. Prod. Rep. 36, 855-868. https://doi.org/10.1039/C9NP00004F

[6] Stuart, K.A., Welsh, K., Walker, M.C., Edrada-Ebel, R., 2020. Metabolomic tools used in marine natural product drug discovery. Expert Opinion on Drug Discovery 15, 499-522. https://doi.org/10.1080/17460441.2020.1722636

[7] Hautbergue, T., Jamin, E.L., Debrauwer, L., Puel, O., Oswald, I.P., 2018. From genomics to metabolomics, moving toward an integrated strategy for the discovery of fungal secondary metabolites. Nat. Prod. Rep. 35, 147-173. https://doi.org/10.1039/C7NP00032D

[8] Olivon, F., Elie, N., Grelier, G., Roussi, F., Litaudon, M., Touboul, D., 2018. MetGem Software for the Generation of Molecular Networks Based on the t-SNE Algorithm. Anal. Chem. 90, 13900-13908. https://doi.org/10.1021/acs.analchem.8b03099

[9] Elie, N., Santerre, C., Touboul, D., 2019. Generation of a Molecular Network from Electron Ionization Mass Spectrometry Data by Combining MZmine2 and MetGem Software. Anal. Chem. 91, 11489-11492. https://doi.org/10.1021/acs.analchem.9b02802

[10] Kauppila, T.J., Syage, J.A., Benter, T., 2017. Recent developments in atmospheric pressure photoionization-mass spectrometry: RECENT DEVELOPMENTS IN APPI-MS. Mass Spec Rev 36, 423-449. https://doi.org/10.1002/mas.21477

[11] Touboul, D., 2019. Is methanol a dopant under atmospheric pressure photoionization conditions? Eur J Mass Spectrom (Chichester) 25, 208-211. https://doi.org/10.1177/1469066718823991

[12] Rhourri-Frih, B., Chaimbault, P., Claude, B., Lamy, C., André, P., Lafosse, M., 2009. Analysis of pentacyclic triterpenes by LC-MS. A comparative study between APCI and APPI. J. Mass Spectrom. 44, 71-80. https://doi.org/10.1002/jms.1472

[13] Zöllner, P., Mayer-Helm, B., 2006. Trace mycotoxin analysis in complex biological and food matrices by liquid chromatography-atmospheric pressure ionisation mass spectrometry. Journal of Chromatography A 1136, 123-169. https://doi.org/10.1016/j.chroma.2006.09.055

[14] Imbert, L., Gaudin, M., Libong, D., Touboul, D., Abreu, S., Loiseau, P.M., Laprévote, O., Chaminade, P., 2012. Comparison of electrospray ionization, atmospheric pressure chemical ionization and atmospheric pressure photoionization for a lipidomic analysis of Leishmania donovani. Journal of Chromatography A 1242, 75-83. https://doi.org/10.1016/j.chroma.2012.04.035

[15] Gaudin, M., Imbert, L., Libong, D., Chaminade, P., Brunelle, A., Touboul, D., Laprévote, O., 2012. Atmospheric Pressure Photoionization as a Powerful Tool for LargeScale Lipidomic Studies. J. Am. Soc. Mass Spectrom. 23, 869-879. https://doi.org/10.1007/s13361-012-0341-y

[16] Zhao, W., Liu, C., Yin, H., Qi, K., Xu, M., Yang, J., Pan, Y., 2019. Direct and rapid analysis of trace levels steroids in water by thermal desorption atmospheric pressure 
photoionization mass spectrometry.

Anal. Methods

11,

1304-1311.

538 https://doi.org/10.1039/C8AY02611D

539

540

541

542

543

544

545

546

547

548

549

550

551

552

553

554

555

556

557

558

559

560

561

562

563

564

565

566

567

568

569

570

571

572

573

574

575

576

577

578

579

580

581

582

583

584

585

586

[17] Arrizabalaga-Larrañaga, A., Rodríguez, P., Medina, M., Santos, F.J., Moyano, E., 2019. Simultaneous analysis of natural pigments and E-141i in olive oils by liquid chromatographytandem mass spectrometry. Anal Bioanal Chem 411, 5577-5591. https://doi.org/10.1007/s00216-019-01939-3

[18] Santerre, C., Vallet, N., Touboul, D., 2018. Fingerprints of flower absolutes using supercritical fluid chromatography hyphenated with high resolution mass spectrometry. Journal of Chromatography B 1092, 1-6. https://doi.org/10.1016/j.jchromb.2018.05.016 [19] Parets, L., Alechaga, É., Núñez, O., Saurina, J., Hernández-Cassou, S., Puignou, L., 2016. Ultrahigh pressure liquid chromatography-atmospheric pressure photoionizationtandem mass spectrometry for the determination of polyphenolic profiles in the characterization and classification of cranberry-based pharmaceutical preparations and natural extracts. Anal. Methods 8, 4363-4378. https://doi.org/10.1039/C6AY00929H

[20] Adeyemi, O.S., Sugi, T., Han, Y., Kato, K., 2018. Screening of chemical compound libraries identified new anti-Toxoplasma gondii agents. Parasitol Res 117, 355-363. https://doi.org/10.1007/s00436-017-5698-1

[21] Sun, X.-L., Tanaka, H., Furuhata, K., SATOSHI OMURA, 1996 Synthesis and Inhibitory Activities of Isochromophilone Analogues against gpl20-CD4 Binding. The Journal Of Antibiotics, 49, 689-692 https://doi.org/10.7164/antibiotics.49.689

[22] Mapari, S.A.S., Thrane, U., Meyer, A.S., 2010. Fungal polyketide azaphilone pigments as future natural food colorants? Trends in Biotechnology 28, 300-307. https://doi.org/10.1016/j.tibtech.2010.03.004

[23] Chen, W., Chen, R., Liu, Q., He, Y., He, K., Ding, X., Kang, L., Guo, X., Xie, N., Zhou, Y., Lu, Y., Cox, R.J., Molnár, I., Li, M., Shao, Y., Chen, F., 2017. Orange, red, yellow: biosynthesis of azaphilone pigments in Monascus fungi. Chem. Sci. 8, 4917-4925. https://doi.org/10.1039/C7SC00475C

[24] Rice-Evans, C.A., Miller, N.J., Paganga, G., 1996. Structure-antioxidant activity relationships of flavonoids and phenolic acids. Free Radical Biology and Medicine 20, 933956. https://doi.org/10.1016/0891-5849(95)02227-9

[25] Celestino, J. dos R., Carvalho, L.E. de, Lima, M. da P., Lima, A.M., Ogusku, M.M., Souza, J.V.B. de, 2014. Bioprospecting of Amazon soil fungi with the potential for pigment production. Process Biochemistry 49, 569-575. https://doi.org/10.1016/j.procbio.2014.01.018 [26] Kumaran, A., Karunakaran, R.J., 2007. Antioxidant activity of Cassia auriculata flowers. Fitoterapia 78, 46-47. https://doi.org/10.1016/j.fitote.2006.09.031

[27] Chambers, M.C., Maclean, B., Burke, R., Amodei, D., Ruderman, D.L., Neumann, S., Gatto, L., Fischer, B., Pratt, B., Egertson, J., Hoff, K., Kessner, D., Tasman, N., Shulman, N., Frewen, B., Baker, T.A., Brusniak, M.-Y., Paulse, C., Creasy, D., Flashner, L., Kani, K., Moulding, C., Seymour, S.L., Nuwaysir, L.M., Lefebvre, B., Kuhlmann, F., Roark, J., Rainer, P., Detlev, S., Hemenway, T., Huhmer, A., Langridge, J., Connolly, B., Chadick, T., Holly, K., Eckels, J., Deutsch, E.W., Moritz, R.L., Katz, J.E., Agus, D.B., MacCoss, M., Tabb, D.L., Mallick, P., 2012. A cross-platform toolkit for mass spectrometry and proteomics. Nat Biotechnol 30, 918-920. https://doi.org/10.1038/nbt.2377

[28] Olivon, F., Grelier, G., Roussi, F., Litaudon, M., Touboul, D., 2017. MZmine 2 DataPreprocessing To Enhance Molecular Networking Reliability. Anal. Chem. 89, 7836-7840. https://doi.org/10.1021/acs.analchem.7b01563

[29] Syage, J.A., 2004. Mechanism of $[\mathrm{M}+\mathrm{H}]+$ formation in photoionization mass

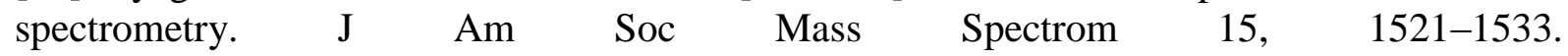
https://doi.org/10.1016/j.jasms.2004.07.006 
[30] Mol, R., de Jong, G.J., Somsen, G.W., 2005. Atmospheric Pressure Photoionization for Enhanced Compatibility in On-Line Micellar Electrokinetic Chromatography-Mass Spectrometry. Anal. Chem. 77, 5277-5282. https://doi.org/10.1021/ac0502697

[31] Kauppila, T.J., Kuuranne, T., Meurer, E.C., Eberlin, M.N., Kotiaho, T., Kostiainen, R., 2002. Atmospheric Pressure Photoionization Mass Spectrometry. Ionization Mechanism and the Effect of Solvent on the Ionization of Naphthalenes. Anal. Chem. 74, 5470-5479. https://doi.org/10.1021/ac025659x

[32] Kostiainen, R., Kauppila, T.J., 2009. Effect of eluent on the ionization process in liquid chromatography-mass spectrometry. Journal of Chromatography A 1216, 685-699. https://doi.org/10.1016/j.chroma.2008.08.095

[33] Robb, D.B., Blades, M.W., 2005. Effects of solvent flow, dopant flow, and lamp current on dopant-assisted atmospheric pressure photoionization (DA-APPI) for LC-MS. Ionizationvia proton transfer. J Am Soc Mass Spectrom 16, 1275-1290. https://doi.org/10.1016/j.jasms.2005.03.017

[34] Zhang, K., Wong, J.W., Mai, H., Trucksess, M.W., 2014. Dopant-Assisted Atmospheric Pressure Photoionization of Patulin in Apple Juice and Apple-Based Food with Liquid Chromatography-Tandem Mass Spectrometry. J. Agric. Food Chem. 62, 4112-4118. https://doi.org/10.1021/jf5006726

[35] Hanold, K.A., Fischer, S.M., Cormia, P.H., Miller, C.E., Syage, J.A., 2004. Atmospheric Pressure Photoionization. 1. General Properties for LC/MS. Anal. Chem. 76, 2842-2851. https://doi.org/10.1021/ac035442i

[36]Watrous, J., Roach, P., Alexandrov, T., Heath, B.S., Yang, J.Y., Kersten, R.D., van der Voort, M., Pogliano, K., Gross, H., Raaijmakers, J.M., Moore, B.S., Laskin, J., Bandeira, N., Dorrestein, P.C., 2012. Mass spectral molecular networking of living microbial colonies. Proceedings of the National Academy of Sciences 109, E1743-E1752. https://doi.org/10.1073/pnas.1203689109

[37] van Santen, J.A., Jacob, G., Singh, A.L., Aniebok, V., Balunas, M.J., Bunsko, D., Neto, F.C., Castaño-Espriu, L., Chang, C., Clark, T.N., Cleary Little, J.L., Delgadillo, D.A., Dorrestein, P.C., Duncan, K.R., Egan, J.M., Galey, M.M., Haeckl, F.P.J., Hua, A., Hughes, A.H., Iskakova, D., Khadilkar, A., Lee, J.-H., Lee, S., LeGrow, N., Liu, D.Y., Macho, J.M., McCaughey, C.S., Medema, M.H., Neupane, R.P., O’Donnell, T.J., Paula, J.S., Sanchez, L.M., Shaikh, A.F., Soldatou, S., Terlouw, B.R., Tran, T.A., Valentine, M., van der Hooft, J.J.J., Vo, D.A., Wang, M., Wilson, D., Zink, K.E., Linington, R.G., 2019. The Natural Products Atlas: An Open Access Knowledge Base for Microbial Natural Products Discovery. ACS Cent. Sci. 5, 1824-1833. https://doi.org/10.1021/acscentsci.9b00806

[38] Gao, J.-M., Yang, S.-X., Qin, J.-C., 2013. Azaphilones: Chemistry and Biology. Chem. Rev. 113, 4755-4811. https://doi.org/10.1021/cr300402y

[38] Son, S., Ko, S.-K., Kim, J.W., Lee, J.K., Jang, M., Ryoo, I.-J., Hwang, G.J., Kwon, M.C., Shin, K.-S., Futamura, Y., Hong, Y.-S., Oh, H., Kim, B.Y., Ueki, M., Takahashi, S., Osada, H., Jang, J.-H., Ahn, J.S., 2016. Structures and biological activities of azaphilones produced by Penicillium sp. KCB11A109 from a ginseng field. Phytochemistry 122, 154-164. https://doi.org/10.1016/j.phytochem.2015.12.008

[40] Takino, M., Daishima, S., Nakahara, T., 2003. Liquid chromatography/mass spectrometric determination of patulin in apple juice using atmospheric pressure photoionization. Rapid Commun. Mass Spectrom. 17, 1965-1972. https://doi.org/10.1002/rcm.1136

[41] Takino, M., Tanaka, T., Yamaguchi, K., Nakahara, T., 2004. Atmospheric pressure photo-ionization liquid chromatography/mass spectrometric determination of aflatoxins in food. Food Additives and Contaminants 21, 76-84. https://doi.org/10.1080/02652030310001632538 